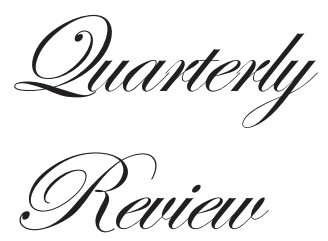

\title{
Portal hypertensive biliopathy: review of pathophysiology and management
}

\author{
Muhammad Rizwan Khan, ${ }_{1}^{1}$ Jibran Tariq, ${ }^{2}$ Rushna Raza, ${ }^{1}$ Muhammad \\ Shahrukh Effendi ${ }^{1}$
}

\section{ABSTRACT}

Department of Surgery ${ }^{1}$ and Medical College, ${ }^{2}$

Aga Khan University and Hospital

Stadium Road, Karachi - 74800

Pakistan

\section{Correspondence:}

Dr. Muhammad Rizwan Khan

Email: doctorrmrkhan@yahoo.com
Portal hypertensive biliopathy is described as abnormalities of the walls of the biliary tree secondary to portal hypertension. In literature it has also been named as "Cholangiopathy associated with portal hypertension", "Portal biliopathy" and "Portal cavernoma associated cholangiopathy". It is usually asymptomatic and found incidentally, but rarely patients do present with symptoms of abdominal pain, jaundice, asthenia and fever. Treatment is warranted in symptomatic cases only, and is dictated by the clinical manifestations and complications of the disease process. Due to presence of underlying severe portal hypertension, endoscopic biliary intervention is usually the first line of management, and is relatively safe and often sufficient. When surgery is resorted to, a porto-systemic shunt prior to biliary bypass procedure provides early relief of obstructive biliary symptoms and often precludes the need for a biliary bypass surgery. This review describes the pathophysiology, presentation, progression and management approaches to portal biliopathy.

KEYWORDS: Portal biliopathy, extrahepatic portal vein obstruction, portal hypertension, biliary stricture

\section{Introduction}

Portal biliopathy has been described as abnormalities arising anywhere in the walls of biliary tree as a result of portal hypertension. ${ }^{1}$ These include intra- and extrahepatic bile duct strictures and dilatation, ischemic injury, choledochal varices and portal cavernoma formation. It is most commonly associated with portal hypertension secondary to portal vein thrombosis. Studies have shown that $80-100 \%$ of patients with extrahepatic portal vein obstruction (EHPVO) manifest clinical or radiological evidence of wall changes typical of portal biliopathy. ${ }^{2}$ Presentation ranges from asymptomatic to obstructive jaundice with or without choledocholithiasis and cholangitis. Recognizing portal biliopathy and its extent and consequences is important so as to follow the correct line of management. The objective of this review is to underline the relevant literature on portal biliopathy with the objectives to highlight areas of pathophysiology and identify various management options for this complex disorder.

\section{Etiology and pathophysiology}

Portal biliopathy shares its etiology with portal hypertension. These include portal vein obstruction, cirrhosis, malignancies, abdominal inflammation, prothrombotic disorders, myeloproliferative disorders and /or congenital hepatic 
fibrosis. ${ }^{3}$ Portal hypertension leads to stagnation of portal blood flow and increases the risk of portal vein thrombosis. The increased pressure in the portal vein causes blood to be diverted through the caval venous system via portosystemic collaterals. Portal cavernoma, bile duct stricture and dilatation and choledochal varices are few manifestations of portal biliopathy resulting from the dilation of these collaterals in the region of porta-hepatis.

Among all the causes of portal hypertension, portal biliopathy is most commonly associated with extrahepatic portal vein obstruction (EHPVO). ${ }^{4}$ Primary EHPVO resulting from omphalitis or umbilical sepsis in childhood usually leads to early onset portal vein thrombosis. The longstanding portal hypertension in this situation leads to manifestation of portal biliopathy in young adulthood. ${ }^{2}$ Dilation of portosystemic collateral vessels at the porta hepatis along with neogenesis of vessels and fibrosis in this region results in the formation of a portal cavernoma, as depicted in Figure 1. The size and complexity of the cavernoma corresponds to the extent of portal vein obstruction. ${ }^{1}$

The venous drainage of common bile duct (CBD) is mainly via two plexus; the epicholedochal venous plexus of Saint and the paracholedochal plexus of Petren. The former is a reticular meshwork of fine venous vessels surrounding the external wall of a bile duct. The paracholedochal plexus of Petren runs along the common bile duct and drains into the gastric, pancreaticoduodenal, portal and hepatic venous system. Dilation of these vessels ( $>1 \mathrm{~mm}$ ) impinges on the smooth mural surface of the ducts. This can be seen on MRCP coupled with MR portography. ${ }^{5}$ In more recent literature, perforators and intracholedochal varices have also been described in patients with portal biliopathy which may have a role in

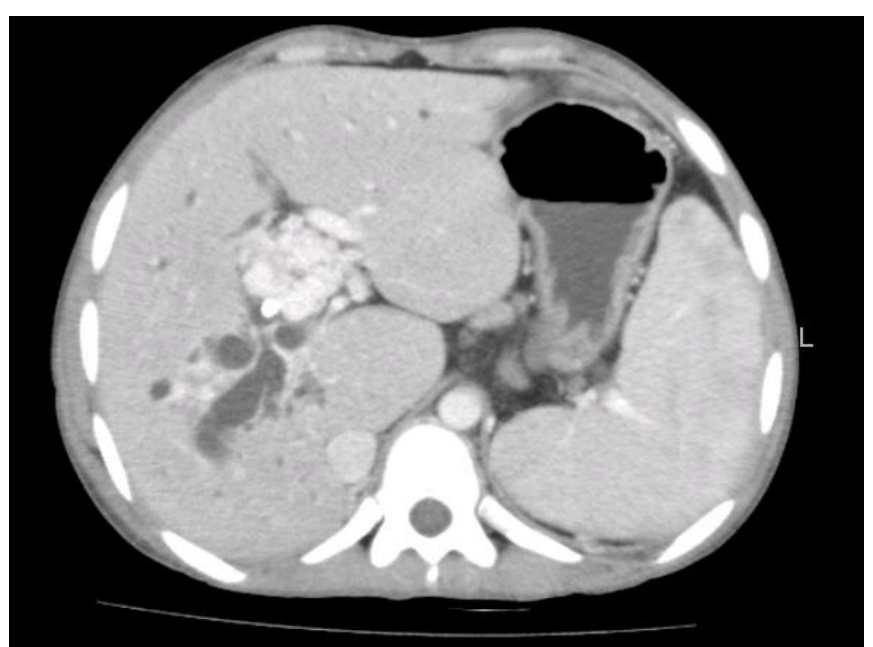

Figure 1: Axial CT scan showing portal vein thrombosis with cavernous transformation pathogenesis of classic features of portal biliopathy. ${ }^{6}$ Ischemia secondary to thrombosis of small venules in bile duct walls has also been proposed as a cause of stricture formations and fibrous scarring at the porta hepatis. Bile duct strictures in some cases of portal biliopathy may also be due to compression by periportal collaterals. ${ }^{7}$

Based on MRCP findings, Shin et $\mathrm{al}^{5}$ classified biliary abnormalities in portal biliopathy into three types; varicoid, fibrotic and mixed. Varicoid irregularities were smooth indentations suggested to be caused by paracholedochal plexus dilatation (cavernous transformation). In the fibrotic type, smooth localized strictures were seen at the level of common bile duct wall thickening. The thickened part of the wall showed delayed increased enhancement on dynamic 3D gradient echo images during MRCP, suggesting an ischemic etiology. In the mixed type, irregular wall contour and thickening were seen, but the thickened wall did not show delayed increased enhancement, suggesting a non-ischemic or inflammatory cause.

\section{Presentation}

Portal biliopathy is usually asymptomatic and is found incidentally during the management of other complications such as esophageal varices. Rarely, it presents with the symptoms of recurrent cholangitis, cholecystitis or choledocholithiasis. ${ }^{8}$ Prospective studies have shown that between $81-100 \%$ of patients with EHPVO have portal hypertensive biliopathy on ERCP, but only a small proportion of these patients are symptomatic. ${ }^{4,9}$ The incidence of symptomatic portal biliopathy ranges from 10 to $38 \%$ in different studies., ${ }^{70-16}$ Cholestasis increases the risk of gall stone disease and therefore can present as obstructive jaundice. The disease may mimic a regional malignancy causing obstructive symptoms. Symptoms such as jaundice, abdominal pain, and fever are associated with higher age, longer duration of disease, higher frequency of gallbladder stones and abnormal liver function tests. ${ }^{7}$ Patient may also present with gastroesophageal or haemmorhoidal bleeding as well as splenomegaly. ${ }^{17}$

\section{Diagnostic tools}

Laboratory findings may reveal elevated bilirubin, alkaline phosphatase and transaminase levels. ${ }^{11}$ Color Doppler ultrasound has been used to demonstrate venous collaterals. 
ERCP has been most helpful in identification of this pathology. ERCP findings reveal extrahepatic and sometimes intrahepatic biliary abnormalities, with the common bile duct being the most common site. The range of findings include strictures, caliber irregularity, segmental upstream dilatation, ectasia, ductal displacement, angulation and pruning of ducts, as shown in Figure 2. ${ }^{11,13}$ The clinical and radiologic presentation can be similar to cholangiocarcinoma ${ }^{14,18}$ or sclerosing cholangitis. ${ }^{4}$ MRCP shows findings similar to ERCP, ${ }^{15}$ and highlights venous collaterals simultaneously as well as being less invasive. When coupled with MR portography, it can differentiate between stones and varices (low T2W signal), and allow better visualization of the biliary tree compared to ERCP. ${ }^{7}$ ERCP is therefore reserved for cases when endoscopic procedure is to be performed concomitantly (Figure 3). However, MRCP has been demonstrated to have a diagnostic pitfall of pseudoobstruction in certain cases. In a study by Watanabe et al, pseudo-obstructions due to artifacts from pulsatile arterial compression were found in 36 of 234 (14\%) patients undergoing MRCP. Most common site was the common hepatic duct followed by the left hepatic duct. ${ }^{19}$ Endoscopic ultrasound (EUS) has also been used to identify pericholedochal varices and bile duct stones. Palazzo et al reported the use of EUS along with Doppler for diagnoses of choledochal varices. ${ }^{20}$ EUS identified choledochal varices in $16(76 \%)$ patients. These

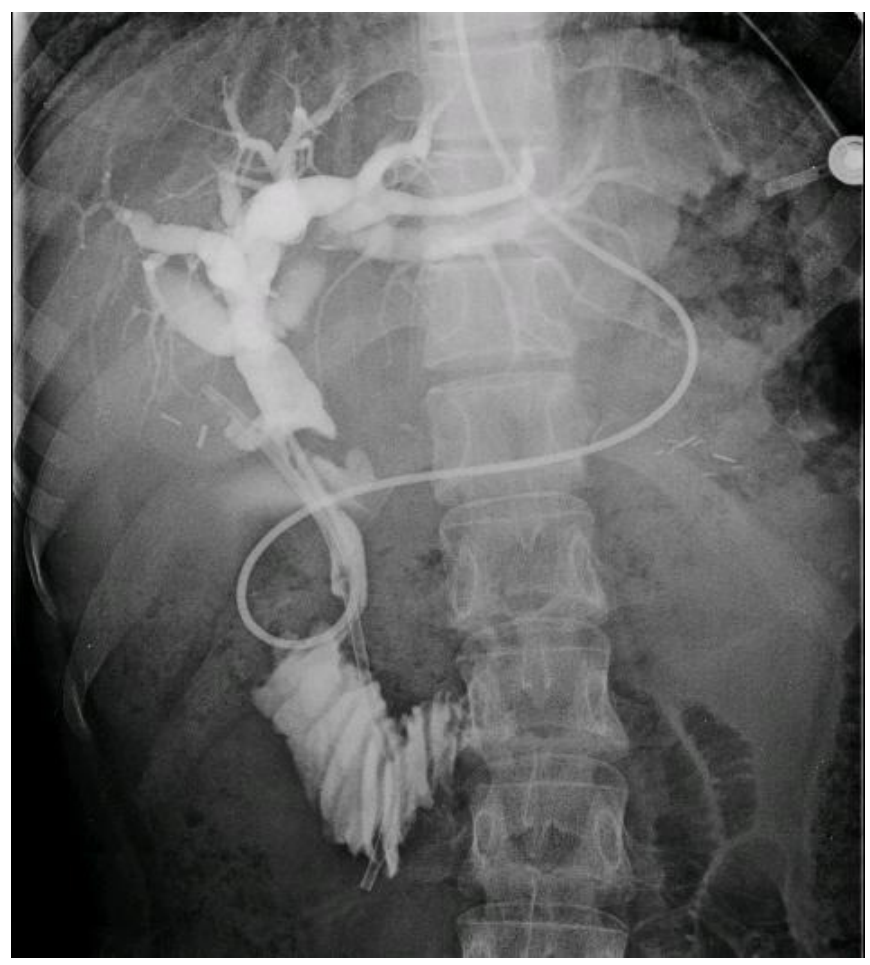

Figure 2: Cholangiogram done through a naso-biliary drain showing classic biliary stricture caused by portal biliopathy

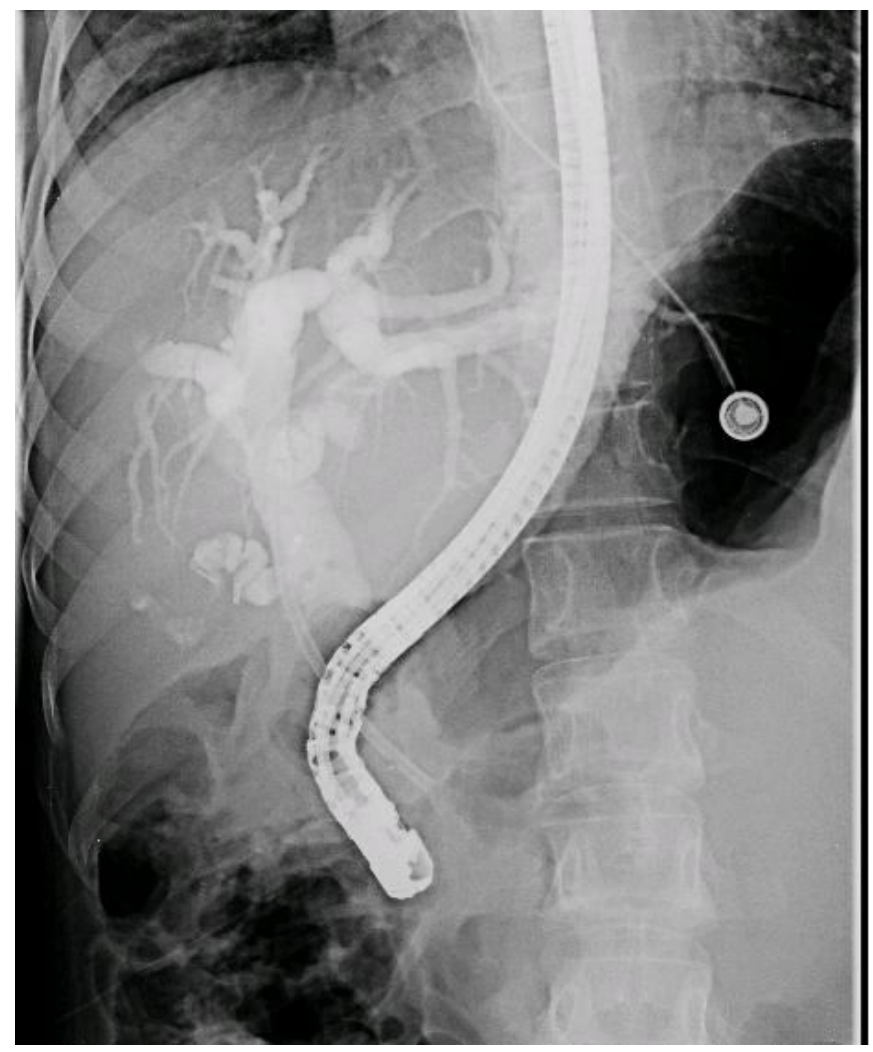

Figure 3: Cholangiogram done through endoscopic retrograde cholangio-pancreatogaraphy (ERCP) showing biliary stricture caused by portal biliopathy. A biliary stent for drainage has been placed.

varices resulted in obstructed jaundice in 3 patients only. Venous channels surrounding the CBD are sometimes very small to be picked up by any other imaging modality.

\section{Management}

Treatment is warranted for symptomatic patients only and should be tailored to each patient's presentation. ${ }^{2,4,11,15}$ This includes relieving symptoms and treating complications arising from biliary obstruction and portal hypertension.?

Different modalities have been described in literature for the management of portal biliopathy. Endoscopic treatment includes biliary stent placement with or without biliary stricture dilatation. Use of nasobiliary drains has also been advocated in such patients. Temporary or permanent endoscopic biliary stenting has been used as the initial therapeutic modality in patients with biliary symptoms. ${ }^{21,22}$ Endoscopic biliary stenting is usually very effective in relieving symptoms of biliary obstruction, but the main disadvantage of stenting is the need for replacement of stent at regular intervals due to blockage or recurrent cholangitis. Repeated stent blockage and incomplete 
drainage of biliary system is an important risk factor for developing secondary biliary cirrhosis. ${ }^{16}$ Dumortier et al reported failure of prolonged endoscopic stent placement in four out of six patients, because of recurrent episodes of cholangitis. Second line decompressive procedure was done in four patients resulting in symptom free survival. ${ }^{23}$ If common bile duct stones develop, they are often recurrent and progressive and may require additional procedures including sphincterotomy, biliary stricture dilation and stone extraction with or without lithotripsy. ${ }^{9,17,24}$ Bleeding from bile duct varices may occur during stones retrieval or bile duct dilatation. Catheter balloon instead of dormia basket have been used in such procedures to avoid bleeding. ${ }^{7}$

Management with endoscopic drainage alone does not always lead to resolution of symptoms and the presence of a stricture may preclude endoscopic treatment completely. Such a situation often requires definitive decompressive surgery. Surgical treatment is also preferred for patients living at far off places, who do not have access to tertiary care centers for frequent stent replacements in case cholangitis or stent blockage occurs. ${ }^{7}$ In patients with endoscopic failure, several studies recommend a two stage procedure; portosystemic shunt (PSS) followed by biliary bypass surgery. ${ }^{1,8,10,17}$ The choice of two-staged procedure also depends on the degree of venous thrombosis as this may render PSS less effective. ${ }^{24}$ Surgery on biliary system with dilated portosystemic collaterals at the porta hepatis can be a significant challenge. Bilio-enteric anastomosis in these patients is associated with high morbidity and mortality because of presence of collateral vessels around the bile ducts, which if injured may cause life threatening haemorrhage. Terlipressin, octreotide or somatostatin can be used to decrease portal pressure effectively during ERCP to reduce risk of hemobilia and before biliary surgery to reduce blood loss in patients without prior shunt surgery. ${ }^{25,26}$ Creation of PSS will allow shunting of blood from the obstructed portal venous system into the systemic venous system. This leads to significantly reduced pressures in the portal vein reducing the size of the cavernoma or the extent of venous collaterals. And subsequent surgery for biliary bypass would be possible with less chances of bleeding.

Porto systemic shunts are divided into selective and nonselective shunts. A selective PSS is usually the preferred surgery chosen to manage portal hypertension. Distal splenorenal shunt (DSRS) is the most commonly employed selective PSS. ${ }^{27,28}$ In this procedure splenic vein is disconnected from the spleno-superior mesenteric vein (SMV) confluence and redirected in to the left renal vein. The coronary vein is ligated to prevent the hepato-fugal flow. A meso-caval shunt may be necessary in circumstances when DSRS is not possible. ${ }^{29}$ This procedure involves use of a graft or a native vein to develop a connection between the inferior vena cava (IVC) and the SMV, below the level of pancreas. The diameter of the shunt is limited to $8 \mathrm{~mm}$ or less in order to preserve some mesenteric flow to the liver.

Portal decompression alone may be sufficient to resolve the symptoms in large proportion of patients. ${ }^{8,23}$ Dhiman et al reported a series of 12 patients with EHPVO and biliary obstruction managed over a period of 10 years. Seven patients had dominant biliary strictures, five out of them underwent shunt surgery, without the need of further biliary bypass. Two patients with biliary strictures and two patients with choledochal varices but without shuntable vein were managed with long term repeated biliary stenting. ${ }^{7}$

Retroperitoneal (splenorenal) approach for creation of a PSS is a better option to avoid the adhesions encountered at subsequent laparotomy for biliary surgery when a transperitoneal approach is used. A transjugular intrahepatic portosystemic shunt may also be feasible in some cases, as it can effectively serve to reduce portal pressure. ${ }^{14,24}$ The Sugiura procedure (gastroesophageal devascularization, transaction and reanastomosis, splenectomy and pyloropasty) has been reserved for more selected cases in the Western world, although it remains the ultimate direct operation for portal hypertension in Japan. ${ }^{30}$ The modified Sugiura procedure is considered an effective rescue surgery in patients unsuitable for selective shunts, transhepatic portosystemic shunt, or transplantation. ${ }^{31}$ This procedure is limited to patients with portosystemic encephalopathy, hepato pulmonary syndrome to control bleeding because of portal hypertension, without creating porto-systemic connection. ${ }^{29}$

Although several studies have shown resolution of biliary strictures with PSS alone, ${ }^{8,17}$ morphologic abnormalities tend to persist as observed in asymptomatic patients with portal cavernoma. ${ }^{13,15}$ Bile duct obstruction may persist because of fibrosis in the pericholedochal areas and stricture formation. These patients require a biliary bypass surgery. ${ }^{8}$ Usually these patients after PSS have a decompressed portal system and portal cavernoma, which makes it possible to construct a bilioenteric anastomosis (hepatico-jejunostomy) proximal to the level of obstruction. But in few cases even after a PSS surgery, there are dilated collaterals surrounding the bile duct, making bilioenteric anastomoses a challenging procedure. ${ }^{25} \mathrm{~A}$ 
surgical bypass operation on a bile duct affected by a portal cavernoma can lead to massive hemorrhage. ${ }^{32}$ Moreover the site of surgical biliary bypass is also an important consideration and dictated by the extent of intrahepatic obstruction by a portal cavernoma as the biliary bypass outlet has to be made at a level higher than the stricture. In such cases where hepaticojejunostomy is not possible, either because of portal cavernoma or because of lengthy/proximal stricture, a "segment III cholangio-jejunostomy" can be a very useful option. This procedure owes much of its popularity to the descriptions made by Blumgart ${ }^{33,34}$ and Bismuth. ${ }^{35}$ This procedure has vastly been used for relieving biliary obstruction due to unresectable hilar cholangiocarcinoma. ${ }^{36,37}$ It includes partially resecting left lobe of liver and making bilioenteric anastomoses using the segment III duct.

A comparative study by Vibert et al depicts better outcome in patients undergoing initial retroperitoneal splenorenal anastomosis followed by bilioenteric bypass surgery. ${ }^{1}$ In this study, most of the patients undergoing a splenorenal shunt had earlier relief of obstructive biliary symptoms and subsequent biliary bypass surgery was required in $50 \%$ of the cases only, which was uneventful. The group managed without PSS required multiple percutaneous or endobiliary procedures to relieve obstructive symptoms, and $44 \%$ of patients still required a surgical bypass which was technically more challenging. The overall outcome in this group was unsatisfactory with recurrent symptoms, need for repeated interventions and one mortality due to severe cholangitis. ${ }^{1}$ In a similar study by Chaudhary et al, seven of nine patients with symptomatic portal biliopathy underwent splenorenal shunting and splenectomy. Jaundice and liver function tests returned to normal in five patients within three to seven weeks. Two patients required a hepaticojejunostomy for persistent biliary symptoms. In these two, LFTs and jaundice returned to normal within two weeks. ${ }^{8}$

These studies provide evidence of success when performing a two staged portosystemic shunting and biliary bypass procedure. On the other hand, D'Souza et al described a case report of portal cavernoma with a tight mid-CBD stricture which was managed initially with a biliary plastic stent. However, the patient presented with recurrent cholangitis, so a proximal splenorenal shunt with splenectomy and cholecystectomy along with the biliary bypass were performed in one stage. Portal blood inflow was controlled by the Pringle maneuver allowing a hepaticojejunostomy created with minimal bleeding. The splenorenal shunt not only decompressed the varices but also reduced bowel edema in the patient, which made the biliary bypass safer. The authors of this case report recommend a single stage portosystemic shunt with biliary bypass when biliary obstruction is caused both by compression by pericholedochal collaterals and biliary stricture. ${ }^{38}$ Although, it may be technically difficult to achieve immediate surgical portal decompression in all cases, the challenges associated with a repeat laparotomy may be avoided by this approach.

Patients with portal biliopathy may also develop gastrointestinal bleeding due to portal hypertension in the perioperative period. Managing these complications with endoscopic variceal ligation or sclerotherapy increases the life expectancy in patients with portal biliopathy. ${ }^{11}$ Liver transplantation may be required if secondary biliary cirrhosis develops or if other procedures are unsuccessful.7,39

\section{Conclusions}

Portal biliopathy is a rare complication of portal hypertension and management is guided by the clinical manifestations and extent of the disease. Endoscopic biliary decompression is the initial modality to be used; however, multiple repeat procedures may be required for effective relief of symptoms. Biliary surgery carries an increased risk of hemorrhage. Therefore when the endoscopic approach fails, it is advisable to perform surgery in two stages; by creating a portosystemic shunt and following it with a biliary bypass surgery. The portosystemic shunt alone may bring relief of symptoms and resolution of biliary strictures, obviating the need for a biliary bypass. Some recent data shows that one stage approach may also be feasible, but further studies are required to evaluate and compare outcomes of one-stage and two-stage procedures. In cases of surgical failure, or where the above approaches cannot be undertaken, liver transplantation may be the only option left.

\section{References}

1. Vibert E, Azoulay D, Aloia T, Pascal G, Veilhan LA, Adam R, et al. Therapeutic strategies in symptomatic portal biliopathy. Ann Surg. 2007;246:97-104.

2. Chandra R, Kapoor D, Tharakan A, Chaudhary A, Sarin SK. Portal biliopathy. J Gastroenterol Hepatol. 2001;16:1086-92.

3. Quarrie R, Stawicki SP. Portal vein thrombosis: What surgeons need to know. OPUS 12 Scientist. 2008;2:30-3.

4. Dilawari JB, Chawla YK. Pseudosclerosing cholangitis in extrahepatic portal venous obstruction. Gut. 1992;33:272-6.

5. Shin SM, Kim S, Lee JW, Kim CW, Lee TH, Lee SH, et al. Biliary abnormalities associated with portal biliopathy: evaluation on 
MR cholangiography. AJR Am J Roentgenol. 2007;188:W341-7.

6. Sharma M Pathak A. Intracholedochal varices in portal hypertensive biliopathy. Eur J Radiol Extra. 2009;72:e119-e23.

7. Dhiman RK, Behera A, Chawla YK, Dilawari JB, Suri S. Portal hypertensive biliopathy. Gut. 2007;56:1001-8.

8. Chaudhary A, Dhar P, Sarin SK, Sachdev A, Agarwal AK, Vij $\mathrm{JC}$, et al. Bile duct obstruction due to portal biliopathy in extrahepatic portal hypertension: surgical management. Br J Surg. 1998:85:326-9.

9. Dhiman RK, Chawla Y, Duseja A, et al. Portal hypertensive biliopathy (PHB) in patients with extrahepatic portal venous obstruction (EHPVO) [abstract]. J Gastroenterol Hepatol. 2006;21:A504.

10. He ZP, Fan LJ. Diagnosis and treatment of portal biliopathy. Hepatobiliary Pancreat Dis Int. 2002;1:581-6.

11. Khuroo MS, Yattoo GN, Zargar SA, Javid G, Dar MY, Khan BA, et al. Biliary abnormalities associated with extrahepatic portal venous obstruction. Hepatology. 1993;17:807-13.

12. Malkan GH, Bhatia SJ, Bashir K, Khemani R, Abraham P, Gandhi MS, et al. Cholangiopathy associated with portal hypertension: diagnostic evaluation and clinical implications. Gastrointest Endosc. 1999;49:344-8.

13. Nagi B, Kochhar R, Bhasin D, Singh K. Cholangiopathy in extrahepatic portal venous obstruction: radiological appearances. Acta Radiol. 2000;41:612-5.

14. Bayraktar Y, Oztürk MA, Egesel T, Cekirge S, Balkanci F. Disappearance of "pseudocholangiocarcinoma sign" in a patient with portal hypertension due to complete thrombosis of left portal vein and main portal vein web after web dilatation and transjugular and intrahepatic portosystemic shunt. J Clin Gastroenterol. 2000;31:328-32.

15. Condat B, Vilgrain V, Asselah T, O'Toole D, Rufat P, Zappa $\mathrm{M}$, et al. Portal cavernoma-associated cholangiopathy: a clinical and MR cholangiography coupled with MR portography imaging study. Hepatology. 2003:37:1302-8.

16. Sezgin O, Oðuz D, Altinta ${ }^{\circ}$ E, Sarita ${ }^{\circ}$ U, Sahin B. Endoscopic management of biliary obstruction caused by cavernous transformation of the portal vein. Gastrointest Endosc. 2003;58:602-8.

17. Khare R, Sikora SS, Srikanth G, Choudhuri G, Saraswat VA, Kumar A, et al. Exrahepatic portal venous obstruction and obstructive jaundice: approach to management. J Gastroenterol Hepatol. 2005:20:56-61.

18. Leclerc JC, Cannard L, Debelle L, Laurent V, Béot S, Régent D. MRI of portal cavernoma with biliary involvement. $J$ Radiol. 2002;83:341-9.

19. Watanabe Y, Dohke M, Ishimori T, Amoh Y, Okumura A, Oda K, et al. Pseudo-obstruction of the extrahepatic bile duct due to artifact from arterial pulsatile compression: a diagnostic pitfall of MR cholangiopancreatography. Radiology. 2000;214:856-60.

20. Palazzo L, Hochain P, Helmer C, Cuillerier E, Landi B, Roseau G, et al. Biliary varices on endoscopic ultrasonography: clinical presentation and outcome. Endoscopy. 2000;32:520-4.

21. Solmi L, Rossi A, Conigliaro R, Sassatelli R, Gandolfi L. Endoscopic treatment of a case of obstructive jaundice secondary to portal cavernoma. Ital J Gastroenterol Hepatol. 1998;30:202-4.
22. Mercado-Díaz MA, Hinojosa CA, Chan C, Anthon FJ, Podgaetz E, Orozco H. Portal biliopathy. Rev Gastroenterol Mex. 2004;69:37-41.

23. Dumortier J, Vaillant E, Boillot O, Poncet G, Henry L, Scoazec JY, et al. Diagnosis and treatment of biliary obstruction caused by portal cavernoma. Endoscopy. 2003;35:446-50.

24. Oo YH, Olliff S, Haydon G, Thorburn D. Symptomatic portal biliopathy: a single centre experience from the UK. Eur $J$ Gastroenterol Hepatol. 2009;21:206-13.

25. Yang JF WX, Li JS, et al. Effect of somatostatin versus octreotide on portal haemodynamics in patients with cirrhosis and portal hypertension. Eur J Gastroenterol Hepatol. 2005;17:53-7.

26. Tyagi P, Sachdeva S, Agarwal AK, Puri AS. Terlipressin in control of acute hemobilia during therapeutic ERCP in patient with portal biliopathy. Surg Laparosc Endosc Percutan Tech. 2009;19:e198-201.

27. Warren WD, Zeppa R, Fomon JJ. Selective trans-splenic decompression of gastroesophageal varices by distal splenorenal shunt. Ann Surg. 1967;166:437-55.

28. Shilyansky J, Roberts EA, Superina RA. Distal splenorenal shunts for the treatment of severe thrombocytopenia from portal hypertension in children. J Gastrointest Surg. 1999;3:167-72.

29. Superina R, Shneider B, Emre S, Sarin S, de Ville de Goyet J. Surgical guidelines for the management of extra-hepatic portal vein obstruction. Pediatr Transplant. 2006;10:908-13.

30. Idezuki Y, Kokudo N, Sanjo K, Bandai Y. Sugiura procedure for management of variceal bleeding in Japan. World J Surg. 1994;18:216-21.

31. Selzner M, Tuttle-Newhall JE, Dahm F, Suhocki P, Clavien PA. Current indication of a modified Sugiura procedure in the management of variceal bleeding. J Am Coll Surg. 2001;193:166-73.

32. Choi HJ, Moon JH, Ko BM, Hong SJ, Koo HC, Cheon YK, et al. Overtube-balloon-assisted direct peroral cholangioscopy by using an ultra-slim upper endoscope (with videos). Gastrointest Endosc. 2009;69:935-40.

33. Blumgart LH, Kelley CJ. Hepaticojejunostomy in benign and malignant high bile duct stricture: approaches to the left hepatic ducts. Br J Surg. 1984;71:257-61.

34. Malt RA, Warshaw AL, Jamieson CG, Hawk JC, 3rd. Left intrahepatic cholangiojejunostomy for proximal obstruction of the biliary tract. Surg Gynecol Obstet. 1980;150:193-7.

35. Bismuth H, Corlette MB. Intrahepatic cholangioenteric anastomosis in carcinoma of the hilus of the liver. Surg Gynecol Obstet. 1975;140:170-8.

36. Chaudhary A, Dhar P, Tomey S, Sachdev A, Agarwal A. Segment III cholangiojejunostomy for carcinoma of the gallbladder. World J Surg. 1997;21:866-70; discussion 70-1.

37. Connor S, Wigmore SJ, Madhavan KK, Parks RW, Garden OJ. Surgical palliation for unresectable hilar cholangiocarcinoma. $H P B$ (Oxford). 2005;7:273-7.

38. D’Souza MA, Desai D, Joshi A, Abraham P, Shah SR. Bile duct stricture due to caused by portal biliopathy: Treatment with one-stage portal-systemic shunt and biliary bypass. Indian $J$ Gastroenterol. 2009;28:35-7.

39. Filipponi F, Urbani L, Catalano G, Iaria G, Biancofiore G, Cioni $\mathrm{R}$, et al. Portal biliopathy treated by liver transplantation. Transplantation. 2004;77:326-7. 